\title{
Los microcréditos para el sector indígena del Cantón Ambato, caso: Incidencia de los microcréditos en el mejoramiento de la calidad de vida del segmento social
} indígena

\author{
Microcredits for the indigenous sector of Canton Ambato, case: Incidence \\ of microcredits in improving the quality of life of the indigenous social \\ segment
}

Mirian Noemí Carranza Guerrero. ${ }^{1}$, Silvia Guadalupe Naranjo Lozada. ${ }^{2}$, Patricia Eugenia Villacrés Jinez. ${ }^{3}$, María Luisa Rodríguez Benavides. ${ }^{4}$

Recibido: 14-08-2020 / Revisado: 18-09-2020 /Aceptado: 22-10-2020/ Publicado: 06-11-2020

\begin{abstract}
.
https://doi.org/10.33262/cienciadigital.v4i4.1.1465

Introduction. The quality of life according to various authors are novel possibilities of life satisfaction, or having a good life in different aspects: social, economic, emotional and educational; Regarding the granting of microcredit, these come to be related to purchasing power. Objective. To investigate how the delivery of microcredits affects the improvement of the quality of life of the indigenous social segment of the towns of the Canton Ambato. Methodology. The research design was based on methods of analysis, theoretical framework through contributions and conceptualizations, descriptive method with the use of the survey and the questionnaire, quantitative and qualitative. The population was 10,973 and the sample of 95 members of the indigenous savings and credit cooperatives of Cantón Ambato. Data was collected through the survey made up of 20 questions, and their interpretation was achieved, identifying how the granting of microcredits affects the quality of life of the indigenous population and determining various aspects that complicate well-being. of the native towns located in the city of Ambato; the contributions and concepts of experts, bibliography and related literature, qualify the study with validity, relevance and coherence. Results. Among the results, we see that the age of the members of the savings and credit cooperatives is 50 years or more, with $43.48 \%$, followed by those between 34 and 41 years old,
\end{abstract}

\footnotetext{
${ }^{1}$ Universidad Técnica de Ambato, Tungurahua Ambato, miriannoemic@ @otmail.com., ORCID: https://orcid.org/0000-0001-5234-8533

${ }^{2}$ Universidad Técnica de Ambato. Tungurahua Ambato, dranaranjo@yahoo.com, ORCID:_https://orcid.org/0000-0003-4559-4060

${ }^{3}$ Instituto Tecnológico Superior "Luis A. Martínez". Tungurahua Ambato, otapgenio1@ @otmail.com, ORCID:_https://orcid.org/0000-0003-3761-6207

4 Instituto Tecnológico Superior “Luis A. Martínez”. Tungurahua, Ambato, luisarob15@yahoo.com ORCID:_https://orcid.org/0000-0002-6950-5969
} 
standing at $22.61 \%$. Regarding marital status, $53.91 \%$ of these are married. The $76.52 \%$ occupation area are merchants. Members have three children and comprise $42.86 \%$. The age of their children is established at 24 years with $14.29 \%$ and it is evident that they work. The number of people who live in each household of the partners is established at $28.75 \%$ with the range of 1 to 3 people, this constitutes that family expenses are not so high and therefore they are subject to credit in a positive way. The partners who have a type of merchant job are registered with $57.14 \%$. The monthly family income is $\$ 1001$ to $\$ 1500$ and corresponds to $42.86 \% .71 .43 \%$ have approved basic education. $90.43 \%$ of affiliates rated the cooperatives very good and good in terms of granting loans quickly and with minimal requirements. The amount of the credits covers $48.70 \%$, with values between $\$ 501$ to $\$ 1000$. The destination they give to the credits obtained is commerce, whose location for the purchase of livestock and a variety of domestic animals is at $41.74 \%$. Compliance with the payment of the monthly payments for the credit granted is set at $83.48 \%$ of partners considered as highly accomplished and accomplished. $74.78 \%$ of cooperators request 1 credit in the 12 months, for financing facilities, payment and minimum requirements. The credit delivery time in 3 days reaches $64.35 \%$ of the partners in an amount less than $\$ 1000$, when this amount exceeds the crediting time is 5 days. The credits requested are mostly for food, livestock, clothing and animal husbandry trade, with $62.61 \%$ of partners. Regarding the type of credit and sex of the affiliates, $62.61 \%$ applied for commercial loans in general and $35.65 \%$ were required by women. $52.78 \%$ of partners are working permanently, an environment where there are mostly women. $100 \%$ of the partners have a business or commercial premises, of which $56.94 \%$ are women as business owners. 91.67\% affirmed that the situation of their businesses improved significantly. Conclusion. It was concluded that the Indigenous Savings and Credit Cooperatives SAC and CREDIPACIFICO have a specific strategic direction, their presence in segment 3 and the provision of their services defines them as icons in the economic / financial sector, as they are fulfilling their objective, which is that of improving the quality of life of the vulnerable sectors of the indigenous population, through the granting of credits and / or microcredits endorsed by the different existing legal protections for this financial segment.

Keywords: Microcredit, Improvement, Quality, Life, Segment, Indigenous.

\section{Resumen}

Introducción. La calidad de vida según varios autores son novedosas posibilidades de satisfacción de vida, o tener una buena vida en los diferentes aspectos: social, económico, emocional y educativo; en lo referente a la concesión de microcrédito, éstos llegan a relacionarse con el poder adquisitivo. Objetivo. Indagar cómo incide la entrega de microcréditos en el mejoramiento de la calidad de vida del segmento social indígena de los pueblos del Cantón Ambato. Metodología. El diseño de investigación se basó en métodos de análisis, marco teórico a través de aportes y conceptualizaciones, método descriptivo con el uso de la encuesta y el cuestionario, 
cuantitativo y cualitativo. La población fue de 10.973 y la muestra de 95 socios de las cooperativas de ahorro y crédito indígenas del Cantón Ambato. Se procedió con la recolección de datos por medio de la encuesta conformada por 20 preguntas, e interpretación de los mismos logrando identificar cómo la concesión de los microcréditos inciden sobre la calidad de vida de la población indígena y determinar variados aspectos que llegan a complicar el bienestar de los pueblos nativos situados en la ciudad de Ambato; los aportes y conceptos de expertos, bibliografía y literatura relacionada, califican al estudio con validez, pertinencia y coherencia. Resultados. Entre los resultados vemos que la edad de los socios de las cooperativas de ahorro y crédito están en los 50 años o más, con el $43.48 \%$ y le siguen los que tienen de 34 a 41 años ubicándose en el $22.61 \%$. Respecto al estado civil el $53.91 \%$ de éstos son casados. El área de ocupación del $76.52 \%$ son comerciantes. Los afiliados tienen tres hijos y abarcan el $42.86 \%$. La edad de sus hijos se establecen en los 24 años con el $14.29 \%$ y se evidencia que ellos trabajan. El número de personas que viven en cada hogar de los socios se establece en un $28.75 \%$ con el rango de 1 a 3 personas, esto constituye que los egresos familiares no son tan altos y por ende son sujetos de crédito de forma positiva. Los socios que tienen un tipo de trabajo de comerciantes están registrados con el 57.14\%. Los ingresos familiares mensuales son de $\$ 1001$ a $\$ 1500$ y corresponde al $42.86 \%$. Tienen educación básica aprobada el $71.43 \%$. El $90.43 \%$ de afiliados calificaron de muy buena y buena a las cooperativas, en cuanto a la concesión de créditos rápidamente y con requisitos mínimos. El monto de los créditos abarca el $48.70 \%$, con valores entre $\$ 501$ a $\$ 1000$. El destino que le dan a los créditos obtenidos es el comercio, cuya ubicación por la compra de ganado y variedad de animales domésticos está en el $41.74 \%$. El cumplimiento de pago de las mensualidades por el crédito otorgado se fija en el $83.48 \%$ de socios considerados como muy cumplidos y cumplidos. El 74.78\% de cooperados solicitan 1 crédito en los 12 meses, por facilidades de financiamiento, pago y requisitos mínimos. El tiempo de entrega del crédito en 3 días llega al $64.35 \%$ de los socios en un monto menor a $\$ 1000$, al superar este monto el tiempo de acreditación es de 5 días. Los créditos solicitados mayormente son para comercio de alimentos, ganado, ropa y crianza de animales, con el $62.61 \%$ de socios. En el tipo de crédito y sexo de los afiliados, $62.61 \%$ solicitaron créditos comerciales en general y el $35.65 \%$ fueron requeridos por mujeres. El $52.78 \%$ de socios están trabajando fijo, entorno donde existen mayormente mujeres. El 100\% de los socios tiene un negocio o local comercial, de cuyo porcentaje el $56.94 \%$ integrado por mujeres como propietarias de los negocios. El $91.67 \%$ afirmaron que la situación de sus comercios mejoró notablemente. Conclusión. Se concluyó que las Cooperativas de Ahorro y Crédito Indígenas SAC y CREDIPACIFICO tienen direccionamiento estratégico determinado, su presencia en el segmento 3 y la prestación de sus servicios las define como íconos en el sector económico/financiero, pues están cumpliendo con su objetivo, que es el de mejorar la calidad de vida de los sectores vulnerables de la población indígena, por medio de la concesión de créditos y/o microcréditos 
avalados por los diferentes amparos legales existentes para este segmento financiero.

Palabras Clave: Microcrédito, Mejoramiento, Calidad, Vida, Segmento, Indígenas.

\section{Introducción.}

El Sistema Integrado de Indicadores Sociales del Ecuador, (SIISE, 2015), registra en Ecuador trece nacionalidades indígenas posicionados en las tres regiones del país, cada una conserva sus propias lenguas y culturas. Los pueblos de la nacionalidad quichua se hallan reconstruyendo su identidad, sistemas de organización y representación que les posibilite desempeñar de forma destacada sus derechos colectivos y asegurar así los escenarios para el progreso a futuro. En los últimos tiempos los hermanos indígenas del Ecuador se han ido transformado en las flamantes figuras de plataformas políticas del país, su presencia en los ámbitos social, político, ideológico e incluso simbólica ha constituido un procedimiento extendido y complicado, que con el transcurrir del tiempo, se han verificado sus resultados, por su gran alta contribución en los diferentes entornos. (SIISE, 2015)

Sobre la calidad de vida, Romero (Romero, 2009), señala que este concepto tiene varios puntos de vista, modalidades, significados, y manera de entenderlo, y emplearlo, pues incluye un elemento objetivo y otro subjetivo. El primero reseña los acuerdos internacionales que hay respecto a la conceptualización de calidad de vida basados en estudios de la Bioética y el otro se corresponde a las diversas maneras de entendimiento personal, y apreciación de las conductas en relación a cada individuo. (Romero, 2009)

En la misma línea argumental, la Organización Mundial de la Salud (OMS, 2006), la calidad de vida la puntualiza como el momento donde se integran el confort físico, cerebral y social de un sujeto, y no solamente la falta de sintomatologías que puedan causarle enfermedades. (OMS, 2006).

Mientras que la Real Academia de la Lengua Española, (RAE, 2001), manifiesta que el termino Vida, proviene del latín Vita que significa fuerza interna fundamental, a través de la que funciona en el ser que la conserva. Igualmente actividad de seres orgánicos; alianza del alma y del cuerpo, lapso que dista del nacimiento de un animal o vegetal hasta su expiración; y subsistencia de las cosas. (RAE, 2001)

En el siguiente texto: la Constitución del Ecuador del año 2008, con base a lo estipulado en el Art. 12, Capítulo Segundo; Derechos del buen vivir; Sección primera Agua y alimentación, señala: El derecho humano al agua es fundamental e irrenunciable. El agua constituye patrimonio nacional estratégico de uso público, inalienable, imprescriptible, inembargable y esencial para la vida. En el Art. 13 Las personas y colectividades tienen derecho al acceso seguro y permanente a alimentos sanos, suficientes y nutritivos; 
preferentemente producidos a nivel local y en correspondencia con sus diversas identidades y tradiciones culturales. El Estado ecuatoriano promoverá la soberanía alimentaria. Y en el Art. 14, se reconoce el derecho de la población a vivir en un ambiente sano y ecológicamente equilibrado, que garantice la sostenibilidad y el buen vivir, sumak kawsay. (Asamblea Nacional, 2008)

Dentro del contexto que se analiza, la Constitución del Ecuador del año 2008, en su Art. 319, reconoce diversas formas de organización de la producción, tales como las comunitarias, cooperativas, empresariales públicas o privadas, asociativas, familiares, domésticas, autónomas y mixtas. El Estado promoverá las formas de producción que aseguren el buen vivir de la población y desincentivará aquellas que atenten contra sus derechos o los de la naturaleza; alentará la producción que satisfaga la demanda interna y garantice una activa participación del Ecuador en el contexto internacional. (Asamblea Nacional, 2008)

La Superintendencia de Economía Popular y Solidaria, mientras tanto en su Ley Orgánica de Economía Popular y Solidaria (LOEPS, 2018), manifiesta que el Sector Financiero Popular y Solidario, se encuentra integrado por instituciones financieras como las cooperativas de ahorro y crédito, entidades asociativas o solidarias, cajas y bancos comunales, y cajas de ahorro. Razón por la cual el sector cooperativo, es considerado el conjunto de cooperativas comprendidas como sociedades de personas que se han fusionado de manera consciente, con el propósito de cubrir sus necesidades de índole económicas, sociales y culturales en común, a través de la conformación de una empresa de propiedad conjunta y de gestión democrática, con personería jurídica, de derecho privado e interés social. Las cooperativas, en su quehacer y relaciones, se someterán a los principios establecidos en la ley, y a valores y principios del cooperativismo universalmente reconocidos. (LOEPS, 2018)

De acuerdo a datos del Banco Central del Ecuador, (BCE, 2019), de entre los socios del cantón Ambato que tienen créditos vigentes en las cooperativas, se ostentan información sobre acceso a la vivienda, calidad de la misma, como materiales de construcción usados en pisos, paredes y techos. Respecto al hacinamiento, número de personas en el hogar, número de cuartos, acceso a servicios sanitarios. Así también, acceso a la educación, asistencia de niños en edad escolar a un establecimiento educativo, edad de los miembros del hogar, asistencia a un establecimiento educativo. La capacidad económica, nivel de ingresos o consumo, costo mínimo de una canasta de bienes y servicios que satisfaga sus necesidades primordiales. Todos estos índices se relacionan con los microcréditos de las cooperativas de ahorro y crédito indígenas. Considerando que las cooperativas de ahorro y crédito constituyen un medio sostenible de llegar a los sectores económicos más vulnerables buscando ser un apoyo, dentro de los cuales se encuentran familias pobres, microempresas, comerciantes informales, emprendedores, individuos de la tercera edad, personas con discapacidades, etc. Además se ha vuelto una de las formas más repetidas entre la población indígena para solicitar microcréditos, pues promueven un apoyo 
considerable entre sus socios o prestamistas, solicitando mínimas garantías financieras, otorgando préstamos en pocos días y a largo plazo, con una tasa de interés baja; todo se basa en valores de ayuda mutua, logrando así el desarrollo comunitario y el mejoramiento de la situación económica de sus afiliados.

La necesidad común que buscan satisfacer las cooperativas de ahorro y crédito indígena, son necesidades de tipo social, como la provisión de artículos de primera necesidad para sujetos de escasos recursos, la resolución a problemas de vivienda, etc. El análisis refleja que en Ecuador existen 887 cooperativas de ahorro y crédito, en Tungurahua se encuentran 117, en el cantón Ambato existen 120, de las cuales 50 son cooperativas de ahorro y crédito indígenas y están reguladas por la Superintendencia de Economía Popular y Solidaria. De acuerdo a la SEPS, y sobre la base de la Resolución JR-STE2012-003 de la Junta de Regulación, las Cooperativas de Ahorro y Crédito, están clasificadas en cuatro segmentos de acuerdo al monto de sus activos, número de socios y sucursales. (BCE, 2019)

El Cantón Ambato tiene diecinueve parroquias, lo cual constituye el 30.1\% del territorio de la provincia de Tungurahua, dispone de 529.856 habitantes, y 51.806 son indígenas, según datos del INEC y Ecuador en cifras y Sistema Nacional de Información. Al hablar de la calidad de vida de la localidad, ésta mejora a través de la búsqueda de la universalización de los derechos mediante la entrega de los servicios de excelencia y calidad y esto se puede lograr con la potencialidad de capacidades de microempresario y emprendimiento; tal objetivo se alcanza el camino a los microcréditos que conceden las cooperativas de ahorro y crédito, a la sociedad que no disponen de recursos, para que emprendan negocios y que ellos prosperen mejorando su estilo de vida, creando riqueza local, con la inyección de capital, adquisición de bienes que le den rentabilidad a sus comercios, lo cual incrementará su poder adquisitivo, tendiente a lograr una vida digna. (INEC, 2020)

Razón demás para que la publicación del Banco Ecuatoriano de la Vivienda, (BEV, 2015), señale que el microcrédito es todo crédito entregado a un prestatario, pudiendo ser alguna empresa constituida; persona natural o jurídica, o un grupo de prestatarios con garantía solidaria, el mismo que tendrá que ser empleado para financiar acciones en pequeña escala, tales como: producción, comercialización o servicios, cuyo origen fundamental de pago a fuente principal de pago es el resultado del producto de la comercialización o ingresos forjados a través de estas diligencias de mercadeo, correctamente comprobados por la institución del sistema financiero prestamista. (BEV, 2015)

Gran importancia contiene la información emitida por el Ministerio de Turismo, a través de la Dirección Provincial de Turismo de Tungurahua, (DPTT, 2013), donde determina que la provincia de Tungurahua acoge a cuatro pueblos indígenas: Chibuleo, Quisapincha, Salasaca y Tomabela; los mismos que por su sistema de vida, logran 
distinguirse con mucha facilidad, pero que también cohabitan y luchan por sus derechos y diferencias reconocidas como tales. (DPTT, 2013)

El autor Alulema (Alulema, 2011), pone de manifiesto en su investigación que el pueblo indígena del cantón Ambato dispone estrategias como son la gestión y el liderazgo cooperativo indígena, mismas que coadyuvan a instaurar instituciones con pleno conocimiento de las circunstancias del medio, para que así se ocupen del bienestar socioeconómico de la sociedad de bajos recursos económicos; entre las más importantes sobresalen las siguientes cooperativas: Mushuc Runa, SAC, Chibuleo, Credipac, Pakarymuy, entre otras, éstas se traducen en una flamante opción basada en la adquisición y desarrollo de nóveles tácticas que le colaboran al sujeto hacia un progreso eficiente y autónomo, mejorando así su calidad de vida.

La estabilidad de las cooperativas de ahorro y crédito indígenas permiten solucionar problemas populares de sectores urbanos marginados del cantón, así como también está encaminada a reactivar segmentos de la producción, pues se evidencia que aproximadamente una cuarta parte de la población indígena no tiene acceso a servicios básicos, como son agua potable, alcantarillado sanitario, electricidad, etc. Así mismo la situación económica con escasos ingresos, pequeño patrimonio y en el peor de los casos ninguno; trabajo independiente para su subsistencia; aspectos que descartan a estas comunidades de la oportunidad de obtener un crédito en el sistema financiero, bancos, instituciones financieras y cooperativas de ahorro y crédito tradicionales y/o con un desarrollo positivo.

Lo expuesto constituye un aspecto negativo que corrobora un nivel de vida con rasgos de pobreza y que se repiten de generación en generación; pero las cooperativas de ahorro y crédito indígenas han conseguido eliminar esta situación, por medio de ciertas deferencias, en relación a las exigencias de ciertos requisitos para la concesión de créditos; siendo las garantías las mínimas a cumplir, entre ellos no solicitar certificado de empleo permanente; es por esto que debido a que acuden al ahorro y crédito cooperativo, se constituye en microempresas de desarrollo económico. (Alulema, 2011)

Tabla 1 Cooperativa de Ahorro y Crédito indígenas

\begin{tabular}{lc}
\hline \multicolumn{1}{c}{$\begin{array}{c}\text { COOPERATIVAS DE AHORRO Y CRÉDITO } \\
\text { INDÍGENAS }\end{array}$} & $\mathrm{N}^{\circ}$ DE SOCIOS \\
\hline SAC & 9.617 \\
CREDIPAC & 1.356 \\
$\mathrm{~N}^{\circ}$ TOTAL DE SOCIOS & 10.973 \\
\hline
\end{tabular}

Fuente: Autoras. Datos extraídos de Alulema, Nelson, 2011 


\section{Metodología.}

En este acápite, se expone la realización de un análisis muy al detalle, respecto a la metodología utilizada, donde se efectúan investigaciones basadas en: Métodos de Análisis: Marco Teórico, por medio de la participación y conceptualizaciones de varios expertos en el área objeto de estudio, documentación que permitió la revisión de la literatura sobre el tema, para así detectar, extraer y recopilar la información de interés, pues la concesión de créditos y microcréditos al sector indígena, por parte de las cooperativas de ahorro, incide de manera radical para el cambio de vida de los pueblos del cantón Ambato, Provincia de Tungurahua; Método Descriptivo que incluye el manejo de la respectiva encuesta para detectar diferentes opiniones, con su instrumento denominado cuestionario; documento que incluye preguntas que deben ser contestadas debidamente para obtener referencias y complementar la investigación; dicha encuesta será aplicada para el acopio de datos directamente de los socios de las distintas cooperativas de ahorro y crédito del sector indígena del cantón Ambato.

La perspectiva del estudio en lo cuantitativo, involucra la confirmación y medición de la bibliografía, así como las evidencias conseguidas para poder ser aplicada en promedios y cálculos estadísticos, por medio de esas herramientas que dan lugar a establecer la confiabilidad de los historiales, para lo cual se llega a determinar una población de 10.973, con una muestra de 95.

Toda esta indagación nos da lugar a conocer el número de microcréditos que realmente son entregados y cómo inciden éstos sobre la calidad de vida de la población indígena, para luego interpretarla haciendo hincapié e impulsando este trabajo en lo que tiene que ver efectivamente al estilo de vida del sector indígena, que se ve reflejado en la inexactitud económica/financiera y social del tema relevante que se explora; todo esto con el ánimo de contribuir a la resolución de los inconvenientes que afectan a seres humanos, que son indígenas pero igual son seres humanos que tienen los mismos derechos, deberes y obligaciones ante la sociedad, ante sí mismos y sus familias. En tanto que en el segmento cualitativo se logró identificar variados aspectos que llegan a complicar la calidad de vida de los pueblos indígenas situados en la ciudad de Ambato, tales como la pobreza que se revela en circunstancias como son: la falta de empleo, ausencia de vivienda propia y adecuada, insuficientes servicios básicos, inaccesibilidad a la educación, falta de planificación familiar, discriminación racial, etc.

Los mencionados habitantes entonces se afianzan en el sistema financiero que aglutina a las cooperativas de ahorro y crédito indígenas, a fin de acceder a microcréditos que les den la oportunidad de salir de la indigencia en la que muchas personas de esta área de la sociedad suelen vivir, para solamente así, librar su lucha constante para conseguir mejores días para sus hijos y familias en general, fundamentados en la posibilidad de crear sus propias pequeñas empresas, mini proyectos, y que generalmente los desarrollan en su propia tierra, como son cultivos, agricultura, avicultura, apicultura, artesanías, 
gastronomía, etc. Se presentan igualmente múltiples secciones y registros constantes en libros, textos, revistas, búsquedas en la web, visitas en el internet e intranet.

\section{Resultados y Discusión}

El estudio muestra síntesis destacadas por lo que resulta muy sugerente enfatizar la exploración efectuada, toda vez que fortalece la importancia que tiene el objetivo sobre la incidencia de los créditos y microcréditos concedidos por parte de las cooperativas de ahorro y crédito indígenas, a estos pobladores del Cantón Ambato, en la búsqueda y mejoramiento de la calidad de vida de los mismos. El análisis se enfoca en las Cooperativas SAC y CREDIPAC, cuyos tipos de créditos se resumen a continuación:

Tabla 2 Cooperativas de Ahorro y Crédito indígenas

\begin{tabular}{|c|c|}
\hline TIPOS DE CRÉDITO & DESTINO \\
\hline Crédito productivo & Financian para adquirir bienes de capital \\
\hline Crédito Comercial Ordinario & $\begin{array}{l}\text { Financian para vehículos livianos, incluyen los con fines } \\
\text { productivos }\end{array}$ \\
\hline Crédito Comercial Prioritario & $\begin{array}{l}\text { Financian para adquirir bienes y servicios para actividades } \\
\text { productivas }\end{array}$ \\
\hline Crédito de Consumo Ordinario & Financian con garantía de naturaleza prendaria o fiduciaria \\
\hline Crédito de Consumo Prioritario & $\begin{array}{l}\text { Financian para compra de bienes y servicios no relativos a la } \\
\text { actividad productiva; otras compras y gastos no del consumo } \\
\text { ordinario }\end{array}$ \\
\hline Crédito Educativo & Financian para su formación y capacitación profesional o técnica \\
\hline Crédito de Vivienda de Interés Público & $\begin{array}{l}\text { Financian con garantía hipotecaria para adquisición o construcción } \\
\text { de vivienda única y de primer uso }\end{array}$ \\
\hline Crédito Inmobiliario & $\begin{array}{l}\text { Financian para comprar bienes inmuebles, construcción de vivienda } \\
\text { de Interés Público, o para reparación, remodelación y mejora de } \\
\text { inmuebles propios. }\end{array}$ \\
\hline Microcrédito & $\begin{array}{l}\text { Financian producción en pequeña escala, con fuente principal de } \\
\text { pago: ventas o ingresos de las actividades. Entrega a persona } \\
\text { natural o jurídica con ventas anuales inferior o igual a } \$ 100000 \text {, o } \\
\text { a grupo de prestatarios con garantía solidaria }\end{array}$ \\
\hline Crédito de Inversión Pública & $\begin{array}{l}\text { Financia programas y proyectos, para la provisión de servicios } \\
\text { públicos, cuya prestación es responsabilidad del Estado, sea } \\
\text { directo, o a través de empresas; y, que se cancelan con recursos, } \\
\text { rentas del deudor fideicomitidas para la entidad financiera pública } \\
\text { prestamista. }\end{array}$ \\
\hline
\end{tabular}

Fuente: Autoras. Datos extraídos de Junta Reguladora del Ecuador, 2015

En la continuación de la búsqueda efectuada sobre el tema, se encuentra lo que acertadamente expone Miño, (Miño, 2013), sobre el hecho de que las cooperativas de ahorro y crédito indígenas constituyen aquellas organizaciones que se destacan en atender los fines sociales, y que sin tener propósitos comerciales o de lucro, su esencia es la 
solidaridad y el mejoramiento económico de sus integrantes, mediante el trabajo en común. (Miño, 2013)

La información general de las cooperativas de ahorro y crédito indígenas se evidencia a partir de diversos campos tales como edad, estado civil, ocupación o profesión, número de hijos, edad de los hijos, número de personas que habitan en el hogar, tipo de trabajo, ingresos familiares mensuales, nivel de educación aprobada, enfermedades, medicina, cirugías, pago de arriendo, accesibilidad a los microcréditos, montos, uso y destino de los recursos, cumplimiento de pagos mensuales, número de créditos, tiempo de entrega del dinero, tipos de créditos y que constan en sus respectivas tablas y figuras explicativas.

Tabla 3 Edad de los socios por cooperativa

\begin{tabular}{lcccccc}
\hline EDAD & \multicolumn{3}{c}{ COOPERATIVAS } & \multicolumn{3}{c}{ TOTAL } \\
\cline { 2 - 7 } & CREDIPAC & \% & SAC & \% & GENERAL & \% \\
$\mathbf{1 8}-\mathbf{2 5}$ & 1 & 0.87 & - & - & 1 & 0.87 \\
$\mathbf{2 6}-\mathbf{3 3}$ & 10 & 8.70 & 9 & 7.83 & 19 & 16.52 \\
$\mathbf{3 4}-\mathbf{4 1}$ & 12 & 10.43 & 14 & 12.17 & 36 & 22.61 \\
$\mathbf{4 2}-\mathbf{4 9}$ & 8 & 6.96 & 11 & 9.57 & 19 & 16.53 \\
$\mathbf{5 0}$ & 23 & 20 & 27 & 23.48 & 50 & 43.48 \\
más & & & & & & \\
\hline
\end{tabular}

Fuente: Autoras. Datos extraídos de COACI, 2015

El nivel de más agrupación está en los 50 años de edad en adelante, con un $43.48 \%$ y son individuos que tienen ingresos fijos y se hallan en posibilidades de solicitar un crédito y dar cumplimiento a los requisitos exigidos por las cooperativas. Los socios entre 18 y 25 años constituyen el porcentaje más bajo, toda vez que son ya que son escasos los socios de ese rango de edad que sí disponen de un trabajo permanente.

Tabla 4 Estado Civil de los socios por cooperativa

\begin{tabular}{|c|c|c|c|c|c|c|}
\hline \multirow{2}{*}{$\begin{array}{c}\text { ESTADO } \\
\text { CIVIL }\end{array}$} & \multicolumn{4}{|c|}{ COOPERATIVAS } & \multicolumn{2}{|c|}{ TOTAL } \\
\hline & CREDIPAC & $\%$ & SAC & $\%$ & GENERAL & $\%$ \\
\hline Soltero & 11 & 9.57 & 17 & 14.78 & 28 & 24.35 \\
\hline Casado & 28 & 24.35 & 34 & 29.57 & 62 & 53.91 \\
\hline Divorciado & - & - & 3 & 2.61 & 3 & 2.61 \\
\hline Viudo & 15 & 13.04 & 7 & 6.09 & 22 & 19.13 \\
\hline
\end{tabular}

Fuente: Autoras. Datos extraídos de COACI, 2015

Predomina el estado civil casado, y se expresa en el $53.91 \%$, lo cual demuestra que el impacto social y económico es apreciable, toda vez que crean sus patrimonios en el núcleo familiar y en estricto apego a las costumbres y tradiciones indígenas, ya que el divorcio es catalogado como una gran lacra e irrespeto hacia la comuna, razón por la que el porcentaje es bajo y equivale al $19.13 \%$. 
Tabla 5 Ocupación / profesión de los socios por cooperativa

\begin{tabular}{lcccccc}
\hline \multicolumn{1}{c}{ OCUPACIÓN } & \multicolumn{3}{c}{ COOPERATIVAS } & \multicolumn{3}{c}{ TOTAL } \\
& CREDIPAC & $\boldsymbol{\%}$ & SAC & $\boldsymbol{\%}$ & GENERAL & $\boldsymbol{\%}$ \\
\hline Comerciante & 41 & 35.65 & 47 & 40.87 & 88 & 76.51 \\
Maquilador & 1 & 0.87 & 2 & 1.74 & 3 & 2.61 \\
Transportista & 3 & 2.61 & 5 & 4.35 & 8 & 6.96 \\
Albañil & 3 & 2.61 & 2 & 1.74 & 5 & 4.35 \\
Empleada & 2 & 1.74 & 4 & 3.48 & 6 & 5.22 \\
Doméstica & & & & & & \\
$\quad$ Maestro & 1 & 0.87 & 1 & 0.87 & 2 & 1.74 \\
Mensajero & 2 & 1.74 & - & - & 2 & 1.74 \\
Cajero & 1 & 0.87 & - & - & 1 & 0.87 \\
\hline
\end{tabular}

Fuente: Autoras. Datos extraídos de COACI, 2015

El área de ocupación de comerciantes, significa la mayor parte de socios de las COACI, con un porcentaje importante que llega al $76.52 \%$; se dedican a la compra y venta de ganado, lácteos, frutas, hortalizas, vestimenta, etc., en los porcentajes cuya tendencia va hacia la baja, se ubican los puestos de cajero, maquilador, maestra, dado que los requerimientos para estas actividades se exige preparación o estudios.

Tabla 6 Número de hijos de los socios por cooperativa

\begin{tabular}{lcccccc}
\hline HIJOS & \multicolumn{3}{c}{ COOPERATIVAS } & \multicolumn{3}{c}{ TOTAL } \\
& CREDIPAC & \% & SAC & \% & GENERAL & \% \\
\hline $\mathbf{1}$ & - & - & 2 & 28.57 & 2 & 28.57 \\
$\mathbf{2}$ & 1 & 14.29 & 1 & 14.29 & 2 & 28.57 \\
$\mathbf{3}$ & 1 & 14.29 & 2 & 28.58 & 3 & 42.86 \\
más de 3 & - & - & - & - & - & - \\
\hline
\end{tabular}

Fuente: Autoras. Datos extraídos de COACI, 2015

El $42.86 \%$ de los socios indicó que tiene 3 hijos y el 57.14\% señaló tener entre 1 y 2 hijos, manifestaron además que si tuvieran mayor número de descendientes, se les hace complejo cubrir la totalidad de los gastos que significa, por lo que ninguno de los socios tiene más de 4 hijos.

Tabla 7 Edad de los hijos de los socios por cooperativa

\begin{tabular}{|c|c|c|c|c|c|c|}
\hline EDAD DE & \multicolumn{4}{|c|}{ COOPERATIVAS } & \multicolumn{2}{|c|}{ TOTAL } \\
\hline $\begin{array}{c}\text { LOS } \\
\text { HIJOS }\end{array}$ & CREDIPAC & $\%$ & SAC & $\%$ & GENERAL & $\%$ \\
\hline 0 - 7 años & - & - & 2 & 28.57 & 2 & 28.57 \\
\hline 8 - 15 años & 1 & 14.29 & 1 & 14.29 & 2 & 28.57 \\
\hline $\begin{array}{l}16-23 \\
\text { años }\end{array}$ & 1 & 14.29 & 1 & 14.29 & 2 & 28.57 \\
\hline $\begin{array}{l}\text { más de } 24 \\
\text { años }\end{array}$ & - & - & 1 & 14.29 & 1 & 14.29 \\
\hline
\end{tabular}

Fuente: Autoras. Datos extraídos de COACI, 2015 
El 57.14\% de los hijos de los socios para cada una de las edades comprendidas entre $0 \mathrm{y}$ 15 años, indicaron que sus hijos son de período escolar, sin constituir sujetos de apoyo económico para el hogar; el 28.57\% comprendidos en edad de 16 a 23 años, trabajan pero sus ingresos nos son fijos, ni estables y tan solamente el $14.29 \%$ exceden los 24 años de edad, no estudian, pero sí trabajan y aportan económicamente a la familia.

Tabla 8 Número de personas en el hogar, por cooperativa

\begin{tabular}{lcccccc}
\hline \multicolumn{1}{c}{$\mathbf{N}^{\circ}$} & & \multicolumn{3}{c}{ COOPERATIVAS } & \multicolumn{3}{c}{ TOTAL } \\
PERSONAS & CREDIPAC & \% & SAC & \% & GENERAL & \% \\
\hline de $\mathbf{1 - 3}$ & - & - & 2 & 28.57 & 2 & 28.57 \\
de $\mathbf{4}$-7 & 1 & 14.29 & 1 & 14.29 & 2 & 28.57 \\
de 7 -10 & 1 & 14.29 & 1 & 14.29 & 2 & 28.57 \\
más de 11 & - & - & 1 & 14.29 & 1 & 14.29 \\
\hline
\end{tabular}

Fuente: Autoras. Datos extraídos de COACI, 2015

Obsérvese en esta pregunta referente al número de personas en el hogar, donde quedó determinado que los socios en el $28.75 \%$ tienen viviendo en su hogar de 1 a 3 personas; lo cual constituye que los egresos familiares no son tan altos, pudiendo satisfacer todo tipo de necesidades de sus miembros filiales; del $28.57 \%$ siguiente habitan entre 4 a 7 personas, lo que demuestra mayores gastos, sin embargo si están en posibilidades de atender todas las obligaciones de la familia; entre 7 a 10 personas residen en ese hogar y significa el $28.57 \%$, lo que viene a causar dificultades para cubrir con los gastos de casa y no se pueden satisfacer todas la situación económica; y el $14.29 \%$ de socios manifestaron que más de 11 personas ocupan su vivienda, esto ya se traduce en un problema para cumplir con los gastos y que deben trabajar doblemente a fin de solventar con lo egresos básicos del hogar, ya que el dinero es un gran limitante, y se traduce en un conflicto al momento de solicitar algún crédito o microcrédito.

Tabla 9 Tipo de trabajo de los socios por cooperativa

\begin{tabular}{lcccccc}
\hline \multicolumn{1}{c}{ TRABAJO } & \multicolumn{3}{c}{ COOPERATIVAS } & \multicolumn{3}{c}{ TOTAL } \\
& CREDIPAC & $\boldsymbol{\%}$ & SAC & \% & GENERAL & \% \\
\hline Albañil & 1 & 14.29 & - & - & 1 & 14.29 \\
Transportista & - & - & 2 & 28.57 & 2 & 28.57 \\
Comerciante & 1 & 14.29 & 3 & 42.86 & 4 & 57.14 \\
\hline
\end{tabular}

Fuente: Autoras. Datos extraídos de COACI, 2015

Los socios de la COACI, en un $57.14 \%$ se desempeñan como comerciantes formales, y mantienen a esta actividad como la primera de su economía; mientras que el $28.57 \%$ son transportistas de cooperativas de buses inter/cantonales, camionetas y taxis, ellos son propietarios de los vehículos en gran parte; y el 14.29\% son albañiles empleados formalmente, y un pequeño porcentaje trabajan de manera independiente y/o informal. 
Los ingresos familiares mensuales del $28.57 \%$ están entre $\$ 501$ a $\$ 1000$; el $42.86 \%$ de los socios tienen entradas de dinero de $\$ 1001$ a $\$ 1500$; el $28.57 \%$ supera los $\$ 1501$ de ingresos familiares, situación que da lugar a que todos los integrantes del núcleo familiar se encuentran en capacidad de trabajar, y así lo hacen para cooperar en la economía del hogar.

Tabla 10 Educación aprobada de los socios por cooperativa

\begin{tabular}{ccccccc}
\hline NIVEL DE & \multicolumn{2}{c}{ COOPERATIVAS } & \multicolumn{3}{c}{ TOTAL } \\
EDUCACIÓN & CREDIPAC & $\%$ & SEC & $\%$ & GENERAL & $\%$ \\
\hline Ninguno & - & - & - & - & - & - \\
Básico & 3 & 42.86 & 2 & 28.57 & 5 & 71.43 \\
Secundaria & 1 & 14.29 & 1 & 14.29 & 2 & 28.57 \\
Superior & - & - & - & - & - & - \\
\hline
\end{tabular}

Fuente: Autoras. Datos extraídos de COACI, 2015

Otra de las preguntas que llama la atención es la número 10, sobre el tipo de educación aprobada por los afiliados y los resultados logrados permiten interpretar que el $71.43 \%$ de los socios solamente tienen educación básica aprobada; el $28.57 \%$ que resta han aprobado la secundaria, aspecto que deja entrever un problema en lo que se refiere al acceso a la educación, por lo que la percepción de los directivos de las cooperativas, es que al no disponer del dinero suficiente para sufragar gastos en planteles particulares, tampoco tendrían para asumir una responsabilidad de pago, razón valedera para que el Estado intervenga urgentemente en este campo social, aplicando estrategias y políticas públicas en beneficio de los ciudadanos que tienen derecho a la educación.

Tabla 11 Accesibilidad de los créditos por cooperativa

\begin{tabular}{lcccccc}
\hline ACCESIBILIDAD & \multicolumn{3}{c}{ COOPERATIVAS } & \multicolumn{3}{c}{ TOTAL } \\
& CREDIPAC & \% & SAC & \% & GENERAL & $\%$ \\
\hline Muy Buena & 29 & 25.22 & 35 & 30.43 & 64 & 55.65 \\
Buena & 22 & 19.13 & 18 & 15.65 & 40 & 34.78 \\
Muy Mala & 2 & 1.74 & 4 & 3.48 & 6 & 5.22 \\
Mala & 1 & 0.87 & 4 & 3.48 & 5 & 4.35 \\
\hline
\end{tabular}

Fuente: Autoras. Datos extraídos de COACI, 2015

Luego de la encuesta aplicada sobre el servicio que brindan las cooperativas, así: adecuada atención por parte del personal, rapidez y agilidad en la entrega del dinero, requisitos mínimos y simples, las respuestas dadas por parte del $90.43 \%$ de los socios se encasillaron en las calificaciones de muy buena y buena; en tanto que el remanente que equivale al mínimo y es el $9.57 \%$ manifestó cierta negatividad en lo que se refiere a las facilidades para obtener créditos. 
Tabla 12 Monto del microcrédito por cooperativa

\begin{tabular}{ccccccc}
\hline MONTO & \multicolumn{4}{c}{ COOPERATIVAS } & \multicolumn{3}{c}{ TOTAL } \\
\cline { 2 - 7 } & CREDIPAC & \% & SAC & \% & GENERAL & \% \\
$\mathbf{1 0 0}-\mathbf{5 0 0}$ & 13 & 11.30 & 16 & 13.91 & 29 & 25.22 \\
$\mathbf{5 0 1}-\mathbf{1 0 0 0}$ & 21 & 18.26 & 24 & 20.90 & 45 & 39.13 \\
$\mathbf{1 0 0 1}-\mathbf{2 0 0 0}$ & 16 & 13.91 & 17 & 14.78 & 33 & 28.70 \\
$\mathbf{2 0 0 1}$ en & 4 & 3.48 & 4 & 3.48 & 8 & 6.96 \\
adelante & & & & & & \\
\hline
\end{tabular}

Fuente: Autoras. Datos extraídos de COACI, 2015

Los créditos y/o microcréditos que solicitan con más asiduidad son de $\$ 501$ a $\$ 1000$, enmarcándose en un porcentaje del $48.70 \%$, que apoyan a los socios a ampliar las ganancias en sus negocios y únicamente el $6.96 \%$ adquiere créditos por montos mayores a \$2000 y que son destinados a consumo, para financiar algún otro tipo de eventos personales, comerciales y hasta para la compra de ganado y/o vehículos.

Tabla 13 Uso del crédito por cooperativa

\begin{tabular}{|c|c|c|c|c|c|c|}
\hline \multirow{2}{*}{$\begin{array}{l}\text { USO DEL } \\
\text { CRÉDITO }\end{array}$} & \multicolumn{4}{|c|}{ COOPERATIVAS } & \multicolumn{2}{|c|}{ TOTAL } \\
\hline & CREDIPAC & $\%$ & SAC & $\%$ & GENERAL & $\%$ \\
\hline $\begin{array}{l}\text { Arreglo de local } \\
\text { comercial }\end{array}$ & 1 & 0.87 & 3 & 2.61 & 4 & 3.48 \\
\hline $\begin{array}{l}\text { Compra de } \\
\text { Ropa }\end{array}$ & 4 & 3.48 & 6 & 5.22 & 10 & 8.70 \\
\hline Cirugías & 4 & 3.48 & 10 & 8.70 & 14 & 12.18 \\
\hline Viajes & 3 & 2.61 & 2 & 1.74 & 5 & 4.35 \\
\hline $\begin{array}{l}\text { Mejoras en la } \\
\text { vivienda }\end{array}$ & 3 & 2.61 & 4 & 3.48 & 7 & 6.09 \\
\hline Estudios & 3 & 2.61 & 2 & 1.74 & 5 & 4.35 \\
\hline Eventos Sociales & 3 & 2.61 & 5 & 4.35 & 8 & 6.96 \\
\hline $\begin{array}{l}\text { Compra de } \\
\text { autos }\end{array}$ & 1 & 0.87 & 3 & 2.61 & 4 & 3.48 \\
\hline $\begin{array}{l}\text { Compra de } \\
\text { ganado }\end{array}$ & 27 & 23.48 & 21 & 18.26 & 48 & 41.74 \\
\hline $\begin{array}{l}\text { Compra de } \\
\text { maquinaria }\end{array}$ & 5 & 4.35 & 5 & 4.35 & 10 & 8.70 \\
\hline
\end{tabular}

Fuente: Autoras. Datos extraídos de COACI, 2015

Una de las actividades económicas principales de los socios, constituye el comercio, cuya ubicación por la compra de ganado y variedad de animales domésticos esté en el 41.74\%; es decir se evidencia que fue el mayor porcentaje de uso que le dieron los socios al dinero del crédito solicitado.

Dentro de estas acciones, los comerciantes no solo realizan la compra venta de ganado, sino que además practican la crianza de animales para su consumo y la venta a la ciudadanía; esto les genera mejores ganancias; otros porcentajes relevantes son para la compra de ropa con el 8.70\%; los socios indicaron que hicieron la adquisición de ropa para el uso diario, para trabajo, eventos varios, y ropa para hacer deporte; en tanto que el $12.17 \%$ estuvo destinado a enfermedades, cirugías, medicinas, etc. 
Tabla 14 Pago de mensualidades por cooperativa

\begin{tabular}{lcccccc}
\hline MENSUALIDADES & \multicolumn{3}{c}{ COOPERATIVAS } & \multicolumn{2}{c}{ TOTAL } \\
& CREDIPAC & \% & SAC & \% & GENERAL & \% \\
\hline Muy Cumplido & 26 & 22.61 & 30 & 26.09 & 56 & 48.70 \\
Cumplido & 22 & 19.13 & 22 & 19.13 & 44 & 38.26 \\
Poco Cumplido & 6 & 5.22 & 6 & 5.22 & 12 & 10.43 \\
Nada cumplido & - & - & 3 & 2.61 & 3 & 2.61 \\
\hline
\end{tabular}

Fuente: Autoras. Datos extraídos de COACI, 2015

Se pudo observar que el $83.48 \%$ de los socios de las COACI, son considerados como muy cumplidos y cumplidos en la cancelación de las mensualidades, según las fechas que le asignó la cooperativa al solicitar el crédito; esto denota que existe una cultura alta y buena de pago. El $16.52 \%$ que resta, se encuentra como poco cumplidos y nada cumplido, dichos clientes tuvieron como justificación para los retrasos en los pagos, debido en su mayor parte al olvido de cancelar las cuotas o por falta de dinero al llegar la fecha señalada, pero que el tiempo de mora no supera los 10 días.

Tabla 15 Número de créditos por cooperativa

\begin{tabular}{ccccccc}
\hline CRÉDITOS & \multicolumn{3}{c}{ COOPERATIVAS } & \multicolumn{3}{c}{ TOTAL } \\
& CREDIPAC & $\boldsymbol{\%}$ & SAC & \% & GENERAL & \% \\
\hline $\mathbf{1}$ & 40 & 34.78 & 46 & 40 & 86 & 74.78 \\
$\mathbf{2}$ & 14 & 12.17 & 15 & 13.04 & 29 & 25.22 \\
\hline
\end{tabular}

Fuente: Autoras. Datos extraídos de COACI, 2015

Los socios de las COACI, en un 74.78\%, han solicitado 1 crédito en los últimos 12 meses, por las facilidades de financiamiento, de pago y requisitos mínimos, entre tanto que solo el $23.22 \%$ de los socios realizaron 2 créditos, revelando que el peor inconveniente es el financiamiento de los créditos, pues al efectuar 2 préstamos, necesariamente el plazo que le financian debe ser más corto.

Tabla 19 Entrega del crédito por cooperativa

\begin{tabular}{lcccccc}
\hline ENTREGA & \multicolumn{3}{c}{ COOPERATIVAS } & \multicolumn{3}{c}{ TOTAL } \\
& CREDIPAC & \% & SAC & \% & GENERAL & \% \\
\hline 3 días & 35 & 30.43 & 39 & 33.91 & 74 & 64.35 \\
5 días & 19 & 16.52 & 22 & 19.13 & 41 & 35.65 \\
\hline
\end{tabular}

Fuente: Autoras. Datos extraídos de COACI, 2015

El porcentaje que llega al $64.35 \%$ de los socios, contaron que en 3 días se les hizo la acreditación a sus cuentas personales, el dinero del crédito solicitado, por ser un monto menor a $\$ 1000$, porque de superar este monto, el tiempo de acreditación es de 5 días. 
Tabla 16 Tipo de crédito por cooperativa

\begin{tabular}{lcccccc}
\hline TIPO DE & \multicolumn{3}{c}{ COOPERATIVAS } & \multicolumn{3}{c}{ TOTAL } \\
CRÉDITO & CREDIPAC & \% & SAC & \% & GENERAL & $\%$ \\
\hline Comercial & 31 & 26.97 & 41 & 35.65 & 72 & 62.61 \\
Consumo & 17 & 14.78 & 12 & 10.43 & 29 & 25.22 \\
Vivienda & 2 & 1.74 & 5 & 4.35 & 7 & 6.09 \\
Educativo & 4 & 3.48 & 3 & 2.61 & 7 & 6.09 \\
& & & & & & \\
\hline
\end{tabular}

Fuente: Autoras. Datos extraídos de COACI, 2015

Los créditos solicitados fueron en su mayoría para actividades inherentes al comercio con el $62.61 \%$, y se derivan en compra - venta de alimentos, ganado, ropa, crianza de animales, etc., también se destinaron en un $25.22 \%$ al consumo, para llevar a cabo heterogéneas actividades tales como cirugías, compra de ropa, maquinaria, reparación de vehículos etc., y la menor cantidad de créditos posicionados fueron para la vivienda y la educación con el $6.09 \%$ ñ cada uno en lo que se refiere a vivienda los montos concedidos no son lo suficientemente altos como para que concreten la construcción de una vivienda, sino que solamente abastecen para mejoras de la misma, y en la educación solamente para financiar cursos de carreras técnicas o especialidades.

Tabla 17 Sexo de los socios y tipo de crédito

\begin{tabular}{cccccccccc}
\hline COMERCIAL & \multicolumn{2}{c}{ CONSUMO } & \multicolumn{2}{c}{ VIVIENDA } & \multicolumn{3}{c}{ EDUCATIVO } \\
\hline F & $\mathbf{6 5}$ & $\mathbf{3 9}$ & $33.91 \%$ & 17 & $14.78 \%$ & 5 & $4.35 \%$ & 4 & $3.48 \%$ \\
M & 50 & 33 & $28.70 \%$ & 12 & $10.43 \%$ & 2 & $1.74 \%$ & 3 & $2.61 \%$ \\
TOTAL & 115 & 72 & $62.61 \%$ & 29 & $\mathbf{2 5 . 2 2 \%}$ & $\mathbf{7}$ & $\mathbf{6 . 0 9 \%}$ & $\mathbf{7}$ & $\mathbf{6 . 0 9 \%}$ \\
\hline
\end{tabular}

Fuente: Autoras. Datos extraídos de COACI, 2015

El $62.61 \%$ de socios han solicitado microcréditos o créditos en general denominados "comercial", de los cuales el $35.65 \%$ son requeridos por mujeres, quienes son dueñas de negocios o locales comerciales. Así también los de "consumo" con el 16.52\%, para "vivienda" 4.35\% y "educativo" el 3.48\%, éstos son los porcentajes más altos en relación al porcentaje total de cada tipo de crédito.

Tabla 18 Sexo de los socios y trabajo

\begin{tabular}{cccccc}
\hline \multicolumn{2}{c}{ SEXO } & \multicolumn{3}{c}{ TRABAJO ACTUAL } \\
\cline { 3 - 6 } & & \multicolumn{3}{c}{ S I } & NO \\
F & $\mathbf{3 8}$ & 38 & $52.78 \%$ & - & - \\
M & $\mathbf{3 4}$ & 34 & $47.22 \%$ & - & - \\
TOTAL & $\mathbf{7 2}$ & $\mathbf{7 2}$ & $\mathbf{1 0 0 \%}$ & - & \\
\hline
\end{tabular}


Se evidencia que el total de los socios, actualmente están trabajando y tienen un trabajo fijo, entorno donde existen más mujeres trabajando.

Tabla 19 Sexo de los socios y local comercial

\begin{tabular}{cccccc}
\hline \multicolumn{2}{c}{ SEXO DE LOS SOCIOS } & \multicolumn{2}{c}{ TIENE UN NEGOCIO O LOCAL COMERCIAL } \\
\multicolumn{3}{c}{ SI } & \multicolumn{2}{c}{ NO } \\
\hline F & $\mathbf{4 1}$ & 41 & $56.94 \%$ & - & - \\
M & $\mathbf{3 1}$ & 31 & $43.06 \%$ & - & - \\
TOTAL & $\mathbf{7 2}$ & $\mathbf{7 2}$ & $\mathbf{1 0 0 . 0 0 \%}$ & - & - \\
& & & & \\
\hline
\end{tabular}

Fuente: Autoras. Datos extraídos de COACI, 2015

El $100 \%$ de los socios tiene un negocio o local comercial, de cuyo porcentaje el $56.94 \%$ está integrado por mujeres y son propietarias de los negocios, mostrando que las féminas indígenas se han vuelto económicamente activas, independientes y se encuentras tomando un empoderamiento en sociedad con su participación.

Tabla 20 Sexo de los socios y situación actual del local comercial

\begin{tabular}{llllll}
\hline $\begin{array}{l}\text { SEXO DE } \\
\text { LOS } \\
\text { SOCIOS }\end{array}$ & \multicolumn{1}{l}{ MEJORÓ SU NEGOCIO O LOCAL COMERCIAL } \\
\hline & & & & & \\
F & $\mathbf{4 1}$ & 40 & $55.56 \%$ & 1 & $1.39 \%$ \\
M & $\mathbf{3 1}$ & 26 & $36.11 \%$ & 5 & $6.94 \%$ \\
$\quad$ TOTAL & $\mathbf{7 2}$ & $\mathbf{6 6}$ & $\mathbf{9 1 . 6 7 \%}$ & $\mathbf{6}$ & $\mathbf{8 . 3 3 \%}$ \\
\hline
\end{tabular}

Fuente: Autoras. Datos extraídos de COACI, 2015

De los socios el $91.67 \%$ afirmaron que existió una mejoría en sus negocios, el $8.33 \%$ no denotó ningún cambio y/o mejoría, siendo el $6.94 \%$ de socios hombres los que no alcanzaron algún tipo de progreso en sus negocios.

\section{Conclusiones.}

- Desde hace algunos años atrás, se viene observando un considerable crecimiento de Cooperativas de Ahorro y Crédito, muchas de ellas cuyas gerencias se encuentran ocupadas por indígenas, principalmente en la provincia de Tungurahua, las mismas que ofrecen una resolución a la situación económica mediante el fomento del ahorro y facilitando crédito y microcréditos a sectores que por mucho tiempo, han sido calificados como poco productivos, extremadamente pobres, ya que sus labores se centraban únicamente en la agricultura, y la transformación del páramo en fructíferos agros de cultivos. 
- En el año 2012 ya se instituye la Superintendencia de Economía Popular y Solidaria; entidad que normaliza el funcionamiento cooperativo del Ecuador, se fundamenta en la Ley Orgánica de Economía Popular y Solidaria y del Sector Financiero Popular y Solidario y su Reglamento que se encuentra en el Decreto Presidencial 1061 conocida como LEPS, la misma que garantiza y asegura tanto los depósitos de los socios, como los créditos de las cooperativas de ahorro y crédito, lo cual es encomiable, ya que a más de cumplir con su propósito esta ley protege la estabilidad en el sector financiero, así como vigila los rendimientos a corto, mediano y largo plazo.

- Las Cooperativas de Ahorro y Crédito Indígenas SAC y CREDIPACIFICO tienen direccionamiento estratégico determinado, su presencia en el segmento tres y por medio de la prestación de servicios las define claramente con su tipo de planeación. Las actividades financieras que entregan a las comunidades indígenas, específicamente los microcréditos, con pocos requisitos y muy accesibles permiten que sean identificadas como íconos en el sector económico/financiero, porque si en algún momento atraviesan alguna cierta debilidad, ésta puede ser superada y mejorable, lo cual no afecta al bienestar de las cooperativas ni de los socios quienes depositan su confianza en dichas instituciones, sino que por el contrario están cumpliendo con el objetivo para el cual fueron creadas, que es el de mejorar la calidad de vida de los sectores vulnerables de la población indígena, por medio de la concesión de créditos y/o microcréditos avalados por los diferentes amparos legales existentes para este segmento financiero.

- Conocer la importancia de los emprendimientos, de las pequeñas y medianas empresas o negocios, permite concluir que los mecanismos que aplican los dueños de este tipo de negocios en ocasiones tienen resultados de poca incidencia, pero la consonancia mediante la que se mueven los negocios demandan cada vez más, plantear nuevos modelos educativos, de gestión, conocimientos, y tecnología.

\section{Referencias Bibliográficas.}

Alulema, N. (2011). IMPACTO SOCIO ECONÓMICO DE LAS COOPERATIVAS DE AHORRO Y. Ambato, Tungurahua, Ecuador. Recuperado el 7 de Febrero de 2020, de repositorio.ute.edu.ec/bitstream/123456789/12157/1/43857_1.pdf

Asamblea Nacional. (2008). En C. d. Ecuador, \& E. Nacional (Ed.). Quito, Pichincha, Ecuador. Recuperado el 22 de Enero de 2020, de https://www.oas.org/juridico/pdfs/mesicic4_ecu_const.pdf

Asamblea Nacional. (2008). Constitución de la República del Ecuador. En E. Nacional (Ed.). Quito, Pichincha, Ecuador. Recuperado el 23 de Enero de 2020, de https://www.oas.org/juridico/pdfs/mesicic4_ecu_const.pdf

BCE. (2019). Estadísticas Monetarias. Boletín Semanal de Tasas de Interés. (E.

Nacional, Ed.) Boletín Semanal BC, pág. 7. Recuperado el 24 de Enero de 2020, de 
https://contenido.bce.fin.ec/documentos/Estadisticas/SectorMonFin/TasasInteres /TasasHistoricoBoletinSemanal.htm

BEV. (2015). Página oficial del Banco de la Vivienda Ecuador. Recuperado el 05 de Febrero de 2020, de http://www.bev.fin.ec/

Constitución del Ecuador. (2008). La Declaración Universal de Derechos Humanos. En LEXIS (Ed.). Quito, Pichincha, Ecuador: Editora Nacional. Recuperado el 17 de Enero de 2020, de https://www.epn.edu.ec/wpcontent/uploads/2015/06/Declaracion-DDHH1.pdf

DPTT. (21 de Enero de 2013). Mitología Tungurahua by Ministerio Turismo. (issuu, Ed.) Recuperado el 5 de Febrero de 2020, de www.turismo.gob.ec

INEC. (Enero de 2020). Ecuador en cifras. (E. Nacional, Editor) Recuperado el 2 de Febrero de 2020, de https://www.ecuadorencifras.gob.ec/estadisticas/

LOEPS. (2018). Ley Orgánica de Economía Popular y Solidaria. En S. d. Solidaria, \& E. Nacional (Ed.). Quito, Pichincha, Ecuador. Recuperado el 26 de Enero de 2020, de http://www.seps.gob.ec/interna-npe?760

Miño, W. (2013). Historia del cooperativismo en el Ecuador. Recuperado el 6 de Febrero de 2020, de www.socioeco.org

OMS. (15 de Marzo de 2006). Calidad de Vida. Recuperado el 15 de Enero de 2020, de http://calidaddevidapabloromero.blogspot.com/

RAE. (2001). Vida. En RAE, Diccionario (Vol. 23). Madrid, Espana. Recuperado el 19 de Enero de 2020, de https://www.rae.es/recursos/diccionarios/diccionariosanteriores-1726-2001/diccionario-de-la-lengua-espanola-2001

Romero, P. (15 de Marzo de 2009). Calidad de Vida. Recuperado el 12 de Enero de 2020, de http://calidaddevidapabloromero.blogspot.com/

SIISE. (27 de Abril de 2015). Sistema Integrado de Indicadores Sociales. (M. C. Social, Editor) Recuperado el 12 de Enero de 2020, de http://www.siise.gob.ec/siiseweb/PageWebs/Marco\%20Conceptual/macsdp_intr od.htm

Sailema A., Maqueira, Sailema, M., \& Sailema, AD., "Estrategia metodológica, esquema corporal y actividad física adaptada: Una trilogía para la inclusión social. Revista Ciencia Digital. ISSN: 2602-8085 Vol. 3, N².5, p. 186-196, abril - junio, 2019.DOI https://doi.org/10.33262/cienciadigital.v3i2.5.53Alonso, G. (2020), Competencias del docente en la atención a estudiantes con conductas disruptivas de 8vo año de Educación General Básica. Tesis en Opción al grado de Magister en Inclusión Educativa y Atención a la Diversidad Universidad Universidad Laica Vicente Rocafuerte. Guayaquil. ( próxima a sustentación)

Campoverde, M. (2014). Desempeño docente en el proceso de enseñanza aprendizaje en el nivel de Educación Básica de la Unidad Educativa "Belisario Quedo" en la ciudad de Pujilí, provincia de Cotopaxi, Año lectivo 2013-2014. Cotopaxi-Ecuador: Universidad Técnico Particular de Loja.

Cépedes, A. (2012). Niños en Pataletas.adolescentes desafiantes, Como manejar los trastornos de conductas en los hijos.Santiago de Chile: Ediciones Básicas Chile SA 
Cuberes, R (2013) Causas de las conductas desafiantes en alumnos de primaria.Trabajo de fin de Grado Universidad Internacional de la Rioja.Facultad de Educación.

Duarte, J. (2017). Formación permanente de docentes en servicio, alternativa para la enseñanza y el aprendizaje de la lengua escrita en la educación básica integral. México: Universidad Rovira.

Freire, C. (2012). Factores que inciden en la presencia de conductas disruptivas de los estudiantes de $\operatorname{los} 8^{\circ}, 9^{\circ} 10^{\circ}$ años en educación básica de la Unidad Educativa experimental FAE N`3 Taura del Cantón Yaguachi durante el período lectivo 2012. Milagro: Universidad Estatal de Milagro.

Flores, G. (2018). Programa de capacitación psicológica a padres de familia para la atención de niños con conductas disruptivas de 6to EGB. Tesis en opción al grado de Magister en Inclusión Educativa y Atención a la Diversidad. Universidad Laica Vicente Rocafuerte. Guayaquil.

Espinoza, G. (2014). Desempeño docente en el proceso de enseñanza aprendzaje en el nivel de Educación Básica Superior del Centro Educativo Colegio de Bachillerato Ciudad de Portovelo. Loja: Universidad Técnica Particular de Loja.

Equipo Dide, 2017, Conductas disruptivas. La asertividad, una competencia docente primordial. http://educaryaprender.es/conductas-disruptivas-comportamiento/

Expasa. (2018). Diccionario Enciclopédico. En T. I.-t. Edición-. Barcelona: Expasa Calpe.

Jiménez, J. (2017). Conductas disruptivas en el Aula y desempeño docentes en la Institución Educativa Técnica General Roberta Leyva del Municipio de Saldaña Tolima. Ibague: Universidad del Tolima.

Jurado, P. (Coord.)(2015), Informe "Influencia de los comportamientos disruptivos en el fracaso escolar de los alumnos de ESO Hacia un modelo de intervención centrado en la institución educativa” Barcelona: UAB (Proyecto I+D-2010. EDU201020105 (subprograma EDUC)

Jurado, P \& Justiano MD(2015), "Las conductas disruptivas y los procesos de intervención en la educación secundaria" Universidad Autónoma de Barcelona (España) Cooperación Técnica Alemana (Bolivia)

Morazán, S. (2013). Competencias docentes y su relación con el rendimiento académico en la asignatura de matemáticas en las instituciones de educación media del municipio de Danlí. Tegucigalpa: Universidad Pedagógica Nacional "Francisco Morazán".

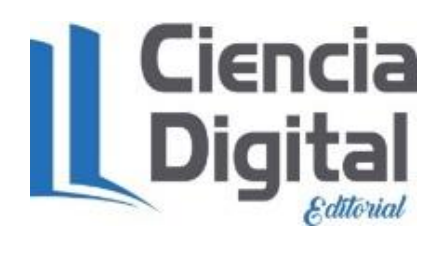




\section{PARA CITAR EL ARTÍCULO INDEXADO.}

Carranza Guerrero, M. N., Naranjo Lozada, S. G., Villacrés Jinez, P. E., \& Rodríguez Benavides, M. L. (2020). Los microcréditos para el sector indígena del Cantón Ambato, caso: Incidencia de los microcréditos en el mejoramiento de la calidad de vida del segmento social indígena. Ciencia Digital, 4(4.1), 81-99. https://doi.org/10.33262/cienciadigital.v4i4.1.1465

\section{LCiencia}

El artículo que se publica es de exclusiva responsabilidad de los autores y no necesariamente reflejan el pensamiento de la Revista Ciencia Digital.

El artículo queda en propiedad de la revista y, por tanto, su publicación parcial y/o total en otro medio tiene que ser autorizado por el director de la Revista Ciencia Digital.
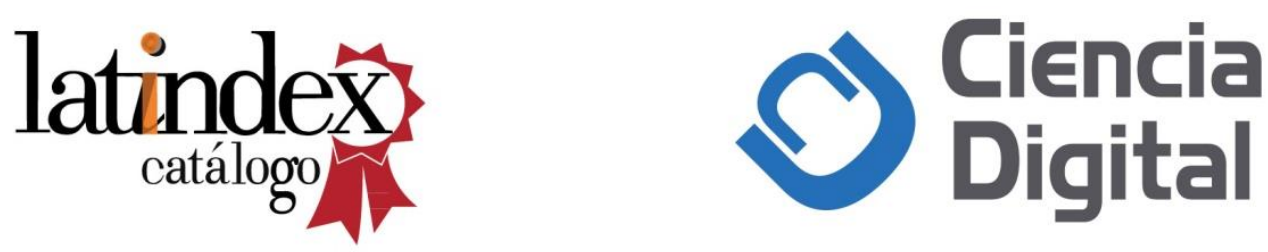